\title{
Vinyl and Linoleum Floorings in Health Infrastructures: Maintenance Recommendations Based on Fieldwork Data
}

\author{
Cláudia Carvalho ${ }^{1}$, Jorge de Brito ${ }^{2}$, Inês Flores-Colen ${ }^{3}$ and Clara Pereira ${ }^{4}$ \\ ${ }^{1}$ Civil Engineer, Instituto Superior Técnico, Universidade de Lisboa, Av. Rovisco Pais, 1049-001 Lis- \\ bon, Portugal, claudia.m.s.carvalho@ist.utl.pt \\ ${ }^{2}$ Full Professor, CERIS, Instituto Superior Técnico, Universidade de Lisboa, Av. Rovisco Pais, 1049- \\ 001 Lisbon, Portugal, jb@civil.ist.utl.pt \\ ${ }^{3}$ Associate Professor, CERIS, Instituto Superior Técnico, Universidade de Lisboa, Av. Rovisco Pais, \\ 1049-001 Lisbon, Portugal, ines.flores.colen@tecnico.ulisboa.pt \\ ${ }^{4} \mathrm{PhD}$ Student, CERIS, Instituto Superior Técnico, Universidade de Lisboa, Av. Rovisco Pais, 1049- \\ 001 Lisbon, Portugal, clareira@sapo.pt
}

\begin{abstract}
Resilient flooring is a popular choice in healthcare facilities, given its durability, comfort, low maintenance and cost. However, anomalies that occur in vinyl and linoleum floorings (VLF) often do not fulfil the expectations of a flooring with good performance and durability. In fact, studies show that pathological phenomena in VLF occur more often in the first years in service, indicating a probable connection with the design and execution stages. These stages are crucial to the quality of VLF, since, if not given adequate attention, pathological symptoms are likely to increase, hence decreasing service life. The authors developed an inspection, diagnosis and rehabilitation system for VLF in healthcare facilities, including: the classification of anomalies, their probable causes, in situ diagnosis methods and repair techniques; anomalies - causes, inter-anomalies, anomalies - diagnosis methods, and anomalies - repair techniques correlation matrices. The same system was validated through the visual inspection of $101 \mathrm{com}-$ partments in six health infrastructures in Lisbon, Portugal, and the respective descriptive statistics analysis of the collected data. That analysis showed, for instance, that the summed contribution of "design errors" and "execution errors" to "faulty welding joints" was 59\%. Making an effort to change the situation diagnosed in the mentioned inspection sample, some design and application stages' recommendations are highlighted, according to the main sensitive issues of the flooring system during its service life. The sensitiveness of VLF highlights the importance of a suitable maintenance plan to minimise susceptibility to various degradation mechanisms.
\end{abstract}

Keywords: Building Pathology, Design and Execution Recommendations, Healthcare Infrastructures, Inspection System, Vinyl and Linoleum Floorings, Maintenance.

\section{Introduction}

Apart from their durability, comfort, low maintenance and cost, vinyl and linoleum floorings (VLF) have ideal properties for health infrastructures, such as static control, slip resistance and antibacterial characteristics. However, without adequate maintenance of VLF, these properties can lose their effectiveness and anomalies may appear, hence decreasing performance levels and life expectancy.

With the recent rise of awareness towards decreasing the ecological footprint of the construction sector, recycling and using more environmental-friendly products have increased. In this context, considering life-cycle assessments, the expectation of extending the service life of the building elements brings new attention to maintenance programs (Ayçam et al., 2017; Harris and Fitzgerald, 
2013). To avoid the development of pathological phenomena in VLF, an adequate maintenance program must be implemented from the beginning of their life-cycle, even before the application of the flooring. Therefore, it is of high importance to study the durability of VLF in sets of buildings in various locations, subject to different actions, in order to understand the pathology according to the characteristics of VLF. Inspections can be systematised and should be included in a maintenance plan, to prevent degradation of the flooring and its substrate.

Regarding the health sector, several studies emphasise the importance of adequate maintenance, in order to prevent hospital-acquired infections (Brito, 2017; Carling et al., 2010; Harris et al., 2010; Harris and Detke, 2013; Harris, 2017). However, apart from maintenance manuals, there is hardly any literature regarding the maintenance of VLF.

An inspection, diagnosis and rehabilitation system for VLF in healthcare facilities (Carvalho et al., 2018) was developed, including: the classification of anomalies, their probable causes, in situ diagnosis methods and repair techniques; anomalies - causes, inter-anomalies, anomalies - diagnosis methods, and anomalies - repair techniques correlation matrices. This system was validated through the visual inspection of 101 compartments in six health infrastructures in Lisbon, Portugal, and the collected data were analysed with descriptive statistics (Carvalho et al., 2019).

The purpose of this paper is to gather the maximum of information available on "Maintenance Recommendations Guides" from several manufacturers, supporting the presented information with the statistical data acquired during the inspection and validation campaign of Carvalho et al. (2018, 2019). Regarding the design and execution stages of VLF, a complete analysis can be found in

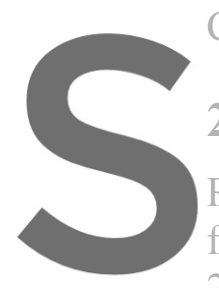
Carvalho et al. $(2018,2019)$.

\section{Installation}

Regarding the installation of

followed. However, there are

2019; Construction, 2013; Ovation, 2013; Poly
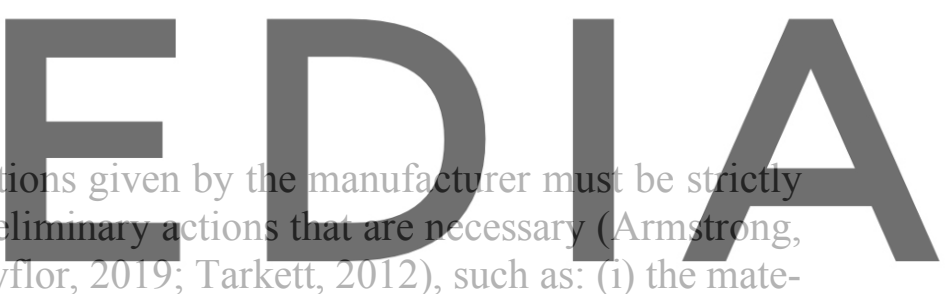

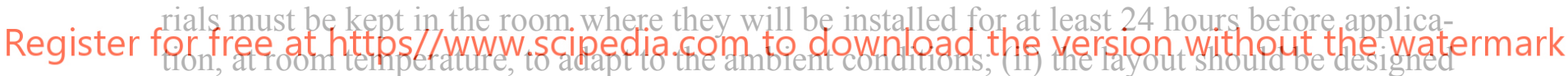

for the lowest number of joints; (iii) the substrate must be dry, clean and level; (iv) if not level,

a levelling screed must be applied; ( $v$ ) before installation, the flooring must be unrolled, measured and pre-cut according to the areas to be coated to minimise waste; (vi) when cutting the material, extra 15-20 cm must be considered, depending on the length; (vii) after the installation of the flooring with adhesives, it will require a drying period, so it should not be walked on, washed or scrubbed within this period.

The preparation of the substrate is the same for vinyl and linoleum, whereas the application varies according to the type of adhesive and drying period. As for the preparation of the substrate, a $10 \mathrm{~cm}$ thick screed is usually applied. However, in rehabilitation works, due to ceiling height limitations, it is not always possible to ensure the minimum screed thickness. In such cases, a glassfibre reinforced screed should be used. Also, on ground floors, a vapour barrier should be applied over the screed.

A levelling compound should be applied over the screed in two cross coats. Silica sand may be added to the second coat to improve adhesion. After drying, any loose sand is vacuumed, the floor is buffed and any dust is cleaned. Next, the coves, with varying heights, usually around $10 \mathrm{~cm}$, are placed on the wall with contact glue. After drying, the pre-cut rolls are placed aligned 
on the floor. The roll is folded in half and the adhesive is carefully applied on the floor (Figure 1a), with a specific comb, and on the coves, with a brush (Figure $1 \mathrm{~b}$ ). To avoid adhesive excess, it must be well spread. The drying period varies and depends on the weather, as humidity will delay drying. After drying, half the roll is placed on the adhesive and the press roller is used.

In order to be well laid, the VLF must be heated, to become flexible and follow the curve of the cove, and then compressed, to ensure adhesion. Therefore, a welding machine is used, without the welding tool, to make the vinyl more ductile (Figure 1c). If this step is skipped, detachment of the coving may occur. In the corners, $45^{\circ}$ cuts must be made (Figure 1d) using a utility knife, which is also used to make straight cuts in vinyl. The curved-tip knife (banana knife) is used to remove excess vinyl on the cove, before fitting it to the upper housing.

Before welding the joints, a half-moon shaped tool is used to create the necessary spacing to place the string. The welding string is then applied using a welding machine or a robot. After the string has cooled, the excess must be removed either by using two tools separately (Figures 1e) or one tool, with a single cut. Then, a transition profile, made of PVC or aluminium, should be placed at each room entrance to ensure a smooth transition between different floorings of similar thickness. Optionally, a silicone string may be applied in the joint between the coving and the wall, to minimise the risk of possible water ingress.

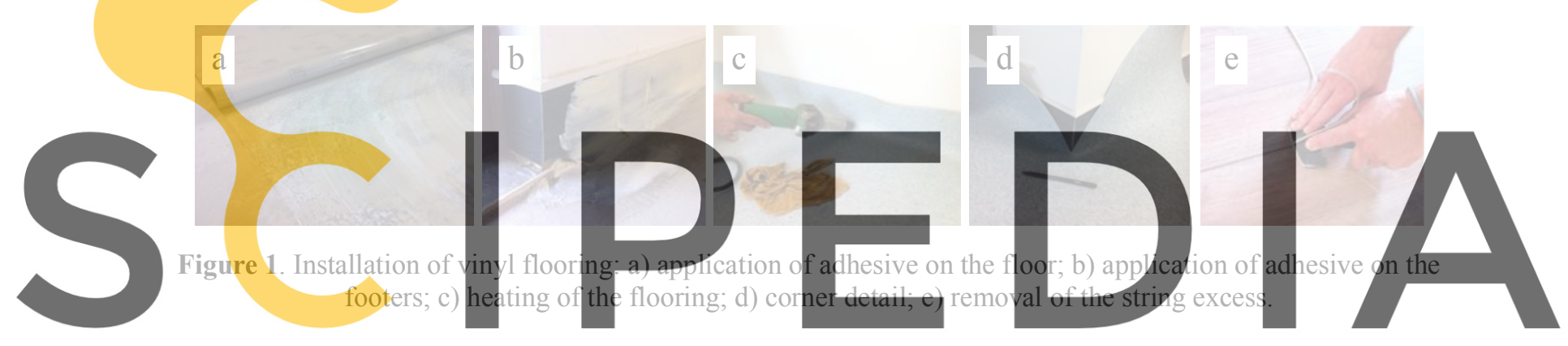

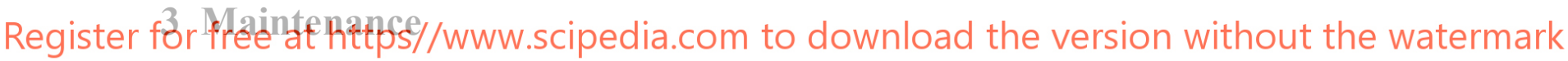
Floorings are selected for many reasons including colour, design and sometimes special properties, such as static control or slip resistance. Without regulair maintenance, dust and soiling build up quickly, changing aesthetic properties and making special features practically useless. Moreover, dirt and soiling usually harbour bacteria, making the flooring a health hazard. Also, dust and grit underfoot can act as an abrasive, which, if left uncontrolled, will shorten the service life of the flooring, causing premature replacement.

Regular and well-planned maintenance keeps the flooring in pristine condition and can enhance its original appearance. It can also reduce wear and ultimately prolong the service life of the flooring. Adequate maintenance (daily/regular and periodic) may lead to high costs in the long run, but the lack of adequate maintenance means higher costs in the future with localised or total replacements. By defining a maintenance programme, real savings can be made without compromising appearance, hygiene and cleanliness requirements.

\subsection{Factor Points to Consider}

Before establishing a maintenance programme, some variables should be considered, as they affect the method, frequency and hence the cost of maintenance. So, the floor location, type and volume 
of traffic, and the existence of dirt barriers should be taken into account. The location of the flooring in the building is one of the main factors, since entrance and reception areas will require more intensive and frequent cleaning than upper floors or low traffic corridors. The types of traffic, density and frequency in the given location also play an important part, since the density of traffic and type of footwear used in common corridors differs from the ones in operation rooms. The expectation of the client for a given location also plays an important part. If a high level of hygiene is required, like in the health department, the maintenance regime must be able to provide it. Finally, the type of cleaning equipment conditions the programme, since manual methods may be time-consuming in large areas and incompatible with the periodicity requirement. However, large machines used in confined spaces can take longer than manual methods (Polyflor, 2019). Additionally, the colour of the flooring is important, since, in general, light colours show soiling more easily and need more frequent cleaning, whereas dark colours more easily show loss of gloss.

\subsubsection{Assessing the location}

The first pant of the maintenance programme process is to break down areas to be cleaned into a series of independent locations. Then, each one should be assessed before a particular maintenance strategy is employed to provide a clear indication as to where the effort and therefore the cost should be applied best.

The assessment should establish the following: type of cleaning; frequency of cleaning; cleaning products and equipment; the level of labour required; and time to be allocated. It is of high importance to regularly assess the different locations and be flexible about the planned maintenance.
If the floor in a particular place needs more attention, then it must be ensured that it is addressed as
soon as possible. On the other hand, if some areas seem over-maintained (e.g. with polish build-up
in the non-walked on areas), then the maintenance level should be adjusted, but the situation must
always be monitored to ensure it remains within control.
According to Carvaltho et al.(2019), anomalies "staining/dirt/colour changes" and "brightness changes" resulted exclusively from causes in groups "environmental actions" and "maintenance

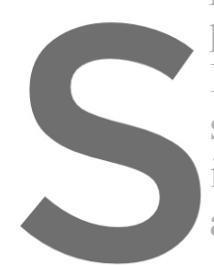

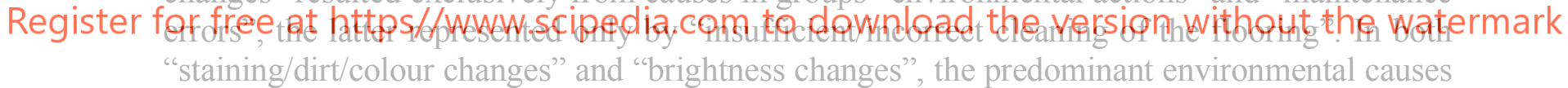
were "biological action/chemically aggressive agents" (50\% and 94\%, respectively). Such frequencies were expected, as the use of inappropriate cleansers or spilling of aggressive substances, such as antiseptic drugs, causes blemishes, changes in brightness, or indelible stains.

\subsubsection{Dirt barrier systems}

The dirt barrier systems (DBS) play an essential role in VLF maintenance, as most dirt, grit and moisture are carried into a building by users. By reducing the amount of these elements that are brought in, not only would this cut the cost of its removal, but it would also cause less abrasive action on the flooring. Also, with less moisture, there would be less potential for slipping. An effective "passive" DBS has both scraping and absorbing qualities and is sufficiently large to perform these actions on both feet during normal walking (Polyflor, 2019).

An exterior and an interior mat should compose the DBS. The exterior scraper mat should be at least two paces long, set into a mat well and have enough height to allow grit to fall below. Inside the building, an interior grade combination of scraper and moisture mat of two to three metres long should be installed, as it removes most moisture and any fine abrasive particles. 
However, to keep the effectiveness of DBS, they must also have adequate maintenance and be cleaned regularly; otherwise, they can increase the soil intake by creating a "soil reservoir" at the entrance of the building.

It is highly important to invest in high-quality floor mats for an extra layer of protection. The mats should be placed at entrances and exits to collect and trap corrosive substances that can be tracked in, such as dirt, sand, oil, grit, asphalt, among others.

\subsection{Types of Maintenance}

The best way to maintain the product characteristics for long-term use is to perform regular cleaning and proper maintenance. In this context, two factors contribute to the decrease of a VLF life-cycle: the lack of maintenance or an inadequate one. The first scenario includes the permanent accumulation of dust, loose dirt and other corrosive materials (such as hand disinfectant). The second scenario includes the use of aggressive cleaning agents, which remove the superficial protection barrier of these floorings, or excessive waxing.

According to Harris (2017), the most common resilient floorings used in patient rooms are rubber, linoleum and vinyl roll and tiles. Tiled vinyl is considered to be the most durable and costeffective at initial costs, though it requires continuous maintenance at high long-term costs. As for vinyl roll, it is less stable and durable than tiles, but its welding joint mitigates the risk of contamination of the bedding layer. Finally, linoleum derives from natural resources, having antistatic and antimicrobial constituents. However, it is susceptible to water damage, usually requiring a surface

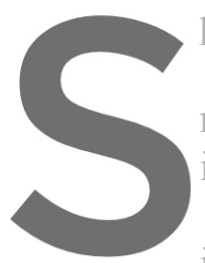
protector that inhibits

\section{Before the flooring} may arise immediately

ing or vacuuming. Also,

Harris and Fitzgerald ings exposed to heavy trat
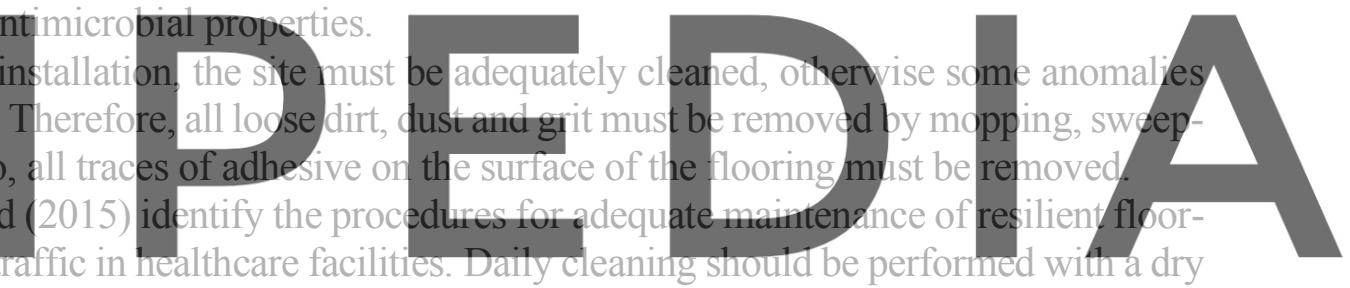

and wet mop; weekly, localised cleaning; quarterly, scrubbing and polishing cleaning; and semi-

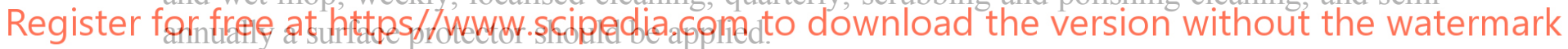

According to Carvalho et al. (2019), anomalies "scratches/wear", "staining/dirt/colour changes"

and "faulty welding joints" occurred in more than half of the inspected floorings. The most frequent anomaly was "scratches/wear", which was expected considering how easy it is to scratch a VLF (Douglas, 2011), since careless dragging of equipment is enough to leave a scratch, and the wheels of most trolleys have inadequate or worn-out material. The second most frequent anomaly is "faulty welding joint". The absence or deficiency of the welding joint, allied with inadequate cleaning, creates a singularity where water may enter, triggering detachment of the flooring. This shows the importance of timely maintenance interventions at welding joints to guarantee the expected service life of the VLF. In general, dirt can be eliminated by cleaning, whereas for indelible stains and colour changes in linoleum floorings, they can only be removed by stripping. In vinyl or recent linoleum floorings, they can be removed by polishing. In these cases, the VLF is only replaced when the affected area is of exceptional aesthetic value.

As for the location of anomalies, in some situations, a specific type of anomaly is predominant. For example, anomaly "scratches/wear" is often seen in poorly executed transition areas or at the rooms' entrance (Figure $3 \mathrm{a}$ and b). It is also the case of anomaly "brightness changes", which often occurs immediately below the alcohol-based disinfectant dispensers (Figure 3c). 
According to Carvalho et al. (2019), there are some repair techniques that should be adopted in the maintenance program, according to the different types of anomalies detected in the regular flooring inspections. For example, the application of a surface protector is a technique widely used for scratches/wear, staining/dirt/colour changes and brightness changes, to reduce its aesthetic impact by smoothing the surface. When scratches exist, partial or total replacement of the flooring should only take place when the area has a high aesthetic value. Stripping is a technique exclusively used in linoleum floorings, being less associated with stains than with brightness changes. In some cases, the floorings are cleaned and waxed regularly, but stripping is only partial and not in the required timings, often resulting in alternated areas with different wax thicknesses, as seen in Figure 3d.
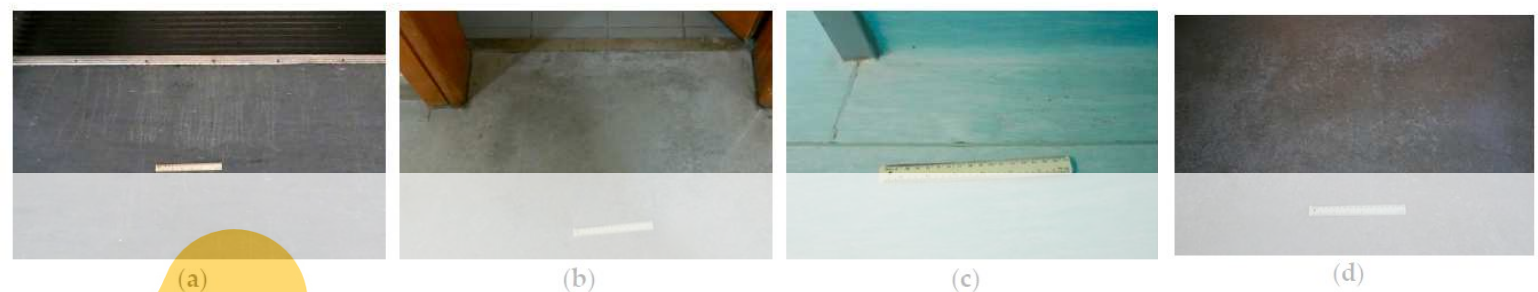

Figure 2. Anomalies; (a) scratches; (b) wear; (c) brightness changes; (d) brightness changes on a linoleum flooring due to inadequate maintenance.

Anomaly prickles/punctures was only not associated with repair techniques "glue injection" and "repair of anomalies affected by the anoma sufficient. For linoleun mixture of white glue a
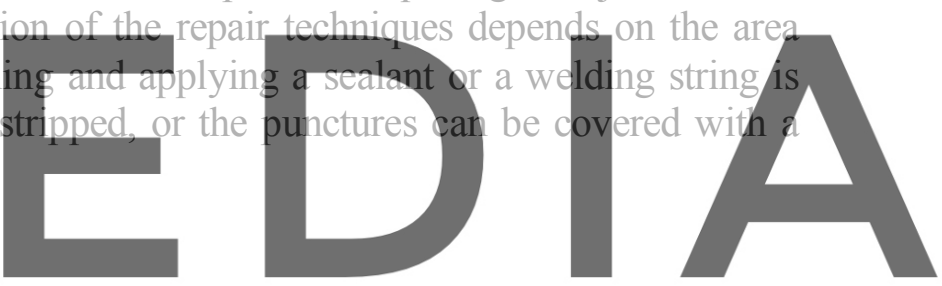

The following recommendations are provided as guidelines. However, the periodicity of each op-

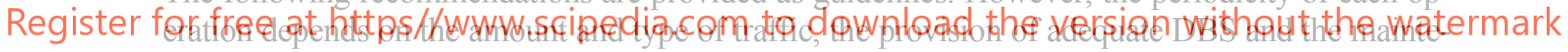
nance equipment available.

For daily vinyi cleaning, the flooring should be mopped, swept or vacuumed to remove dust and loose dirt. Stains can be easily removed with detergent and subsequently rinsed. If required, to remove persistent black marks, the floor may be damp mopped with a neutral cleanser-the label must always be read to ascertain suitability, and the manufacturer's instructions for application must be followed. It is highly important that the flooring is washed with nonabrasive cleaners. When rinsed, the floor must be allowed to dry. All smooth floorings can be slippery when wet. If necessary, the floor may be buffed with a dry soft mop or cloth. The appearance of the flooring should be assessed weekly.

Once a week or, if necessary, more often, the floor should be cleaned with a damp cloth or a mop soaked in warm water and a neutral cleaner. Light scuffing is executed with a buffing machine with a suitable dry and clean pad.

\subsubsection{Periodic maintenance}

The appearance of the floor should be periodically assessed. If the floor has dirt build-up, it should be machine scrubbed with a scrubber dryer fitted with a suitable clean pad, using neutral detergent. 
Then, it should be rinsed thoroughly and allowed to dry. To restore the finish, it may be dry buffed.

Regarding the stripping and resealing of a linoleum floor, according to Gorrée et al. (2002), stripping should be done six times in 20 years (approximately every three years) in public buildings.

\subsubsection{Preventive measures}

To prevent damage, some precautions should be taken. For example, spills should be cleaned immediately, since the longer spilt materials are left on the floor, the higher the risk of irreversible staining. Regarding the furniture, it should always be lifted and carried, instead of pushed, pulled, or dragged over the floorings. According to Carvalho et al. (2019), cause "dragging of equipment" was the most frequent, being indicated in approximately $28 \%$ of anomalies, probably due to a close correlation with the occurrence of scratches. This cause represents the use of improper wheel material, which easily leaves a mark in VLF, or moving of furniture without adequate protections. Furthermore, heavy furniture or appliances that are not moved often should be equipped with flat, non-staining floor protectors, to avoid indentation from heavy furniture or sharp furniture feet. The heavier the furniture, the wider the protectors should be (Seeley, 1985). Vacuum cleaners with beater bars should be avoided, as they can damage the flooring. Black rubber wheels and rubber feet can cause discolouration. Strong detergents must be avoided, as they may wear the protective layer. Cleaning and maintenance training courses from manufacturers, including both practical and theory work, should help facilities maintenance staff get the best from vinyl floorings by using adequate cleaning methods and products

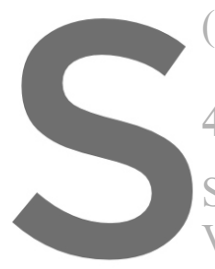
(Polyflor, 2019; Tarketr)

4 Conclusions and

Several difficulties were
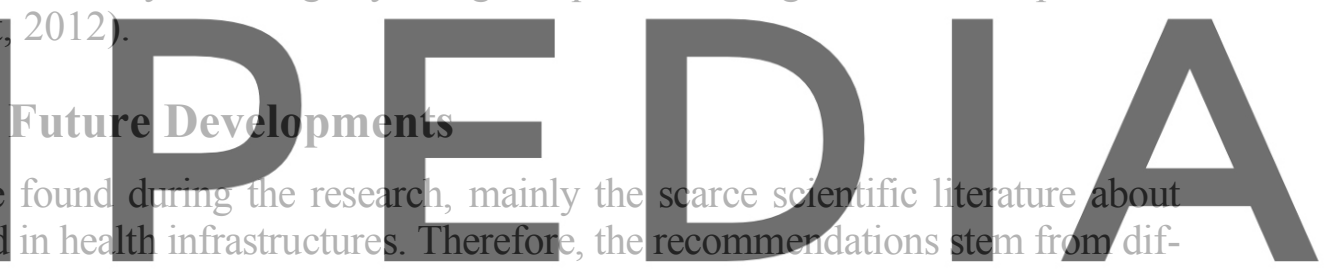

ferent sources, namely supplier guidelines, site assessment and statistical evaluation from site as-

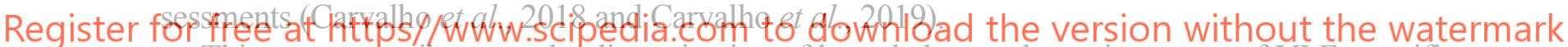
This paper contributes to the dissemination of knowledge on the maintenance of VLF, specifically in health infrastructures, as the presented information may be used to improve and increase the effectiveness of maintenance plans. Additionally, it may also be useful at the application stage, as some defects with substrate origin, for instance, may be prevented with adequate cleaning of the substrate. Moreover, at the design stage, a maintenance manual or a concise user's manual of the flooring may be developed and delivered to the client. Furthermore, the consideration of efficient dirt barriers, with scraping and absorbing qualities, should take place early at the specification stage, and not afterwards, when there are rarely enough funds or space to do the job correctly.

To avoid defects at the application stage, several measures may be implemented, always starting with the use of specialised labour and better communication between contractors. Specialised labour is more likely to apply floorings according to the best practices, hence avoiding defects caused by execution errors. In terms of maintenance, regular cleaning is more beneficial and cost-effective to the flooring than occasional heavy cleaning.

Regarding future developments to increase the life cycle, cleaning and maintenance training courses given by the manufacturers should be mandatory. It is essential to train the cleaning staff since inadequate maintenance directly contributes to accelerating the degradation of VLF. Moreover, regular inspections are also necessary to ensure the quality control of the VLF application. It is 
also expected that the efficiency and effectiveness of maintenance in VLF increase if the inspection system proposed by Carvalho et al. (2018) is used. In the future, to improve the system, a wider inspections campaign should be made.

\section{Acknowledgements}

The authors gratefully acknowledge the support of the CERIS Research Institute; IST, University of Lisbon; and FCT, Foundation for Science and Technology, through the BestMaintenance Project PTDC/ECI-CON/29286/2017.

\section{ORCID}

Cláudia Carvalho: https://orcid.org/0000-0002-0804-6594

Jorge de Brito: https://orcid.org/0000-0001-6766-2736

Inês Flores-Colen: https://orcid.org/0000-0003-4038-6748

Clara Pereira: https://orcid.org/0000-0002-9535-1844

\section{References}

Agence Qualité Construction (2013). Fiche no F1 - Décollement de revêtements de sols souples collés.

Armstrong: "Luxury Vinyl Tile a \& plank maintenace recommendations" (website:

https://www.armstrongflooring.com/pdbupimages-flr/205123.pdf, visited on October 2019);

Ayçam, I. and Yazici, A. (2017). Evaluation of operating room units within the context of green design criteria. In GU J. Sci., 30, 1-15.

Brito, F. (2017). Controlo da contaminação de superfícies no meio hospitalar (Contamination control of surfaces in the hospital environment). TecnoHospital, 79, 10-11 (in Portuguese).

Carling, P.C. and Bartley,

can harm your patient

Carvalho, C., de Brito, J. for vinyl and linoleum flo

Carvalho, C., de Brito, J., F Floorings in Health Infras

Douglas, J. and Noy, E.A. (2011). Building Surveys and R
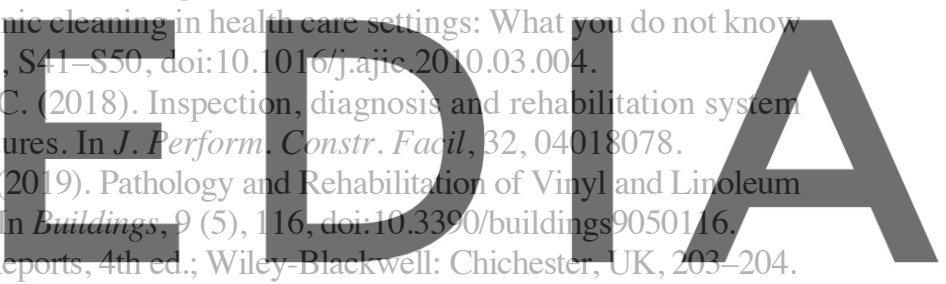

Gorrée, M., Guinée, J.B., Huppes, G. and Oers, L.V. (2002). Environmental life cycle assessment of linoleum. Int.

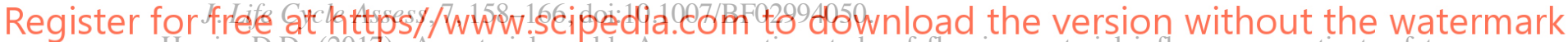

Harris, D.D. (2017). A material world: A comparative study of flooring material influence on patient safety, satisfaction, and quality of care. J. Interior Des. 42, 85-104; doi:10.1111/joid.12100.

Harris, D.D., Pacheco, A. and Lindner, A.S. (2010). Detecting potential pathogens on hospital surfaces: An assessment of carpet tile flooring in the hospital patient environment. In Indoor Built Environ. 19, 239-249, doi:10.1177/1420326X09347050.

Harris, D.D. and Detke, L.A. (2013). The role of flooring as a design element affecting patient and healthcare worker safety. HERD-Health Environ. Res. 6, 95-119, doi:10.1177/193758671300600308.

Harris, D.D. and Fitzgerald, L. (2013). A life-cycle cost analysis for flooring materials for healthcare facilities. In J. Hosp. Adm., 4, 92-100, doi:10.5430/jha.v4n4p92.

Lent, T., Silas, J. and Vallete, J. (2010). Chemical hazards analysis of resilient flooring for healthcare. HERDHealth Environ. Res., 3, 97-117, doi:10.1177/193758671000300209.

Ovation Interior Flooring Design Limited: "Care and maintenance instructions for vinyl floors", 1 (4) 2013-01-01 (website:http://www.ovationflooring.com/wp-content/uploads/2015/11/Oceanic_Maintenance_Info.pdf, visited on October 2019);

Polyflor: "Floor Care and Maintenace", Section 14 (website: https://www.polyflor.ca/maintenance-guides, visited on October 2019);

Seeley, I. H. (1985). Building Surveys, Reports and Dilapidations. Macmillan;

Tarkett: "Cleaning and Maintenace Guides" for iQ Range, Homogeneous compact vinyl, April 2012. 